\title{
Testing, characterization and modelling of mechanical behaviour of poly (lactic-acid) and poly (butylene succinate) blends
}

\author{
T. Y. Qiu', M. Song ${ }^{2}$ and L. G. Zhao ${ }^{1 *}$
}

\begin{abstract}
Background: Significant amount of research, both experimental and numerical, has been conducted to study the mechanical behaviour of biodegradable polymer PL(L)A due to its wide range of applications. However, mechanical brittleness or poor elongation of PL(L)A has limited its applications considerably, particularly in the biomedical field. This study aims to study the potential in improving the ductility of PLA by blending with PBS in varied weight ratios.

Methods: The preparation of PLA and PBS blends, with various weight ratios, was achieved by melting and mixing technique at high temperature using HAAKE ${ }^{\text {TM }}$ Rheomix OS Mixer. Differential Scanning Calorimetry (DSC) was applied to investigate the melting behaviour, crystallization and miscibility of the blends. Small dog-bone specimens, produced by compression moulding, were used to test mechanical properties under uniaxial tension. Moreover, an advanced viscoplastic model with nonlinear hardening variables was applied to simulate ratedependent plastic deformation of PLA/PBS blends, with model parameters calibrated simultaneously against the tensile test data.
\end{abstract}

Results: Optical Microscopy showed that PBS composition aid with the crystallization of PLA. The elongation of PLA/PBS blends increased with the increase of PBS content, but with a compromise of tensile modulus and strength. An increase of strain rate led to enhanced stress response, demonstrating the time-dependent deformation nature of the material. Model simulations of time-dependent plastic deformation for PLA/PBS blends compared well with experimental results.

Conclusions: The crystallinity of PLA/PBS blends increased with the addition of PBS content. The brittleness of pure PLA can be improved by blending with ductile PBS using mechanical mixing technique, but with a loss of stiffness and strength. The tensile tests at different strain rates confirmed the time-dependent plastic deformation nature of the blends, i.e., viscoplasticity, which can be simulated by the Chaboche viscoplastic model with nonlinear hardening variables.

Keywords: PLA/PBS blends, Mixing, Mechanical properties, Crystallization, Viscoplastic model

\footnotetext{
* Correspondence: L.Zhao@Lboro.ac.uk

${ }^{1}$ Wolfson School of Mechanical, Electrical and Manufacturing Engineering,

Loughborough University, Loughborough LE11 3TU, UK

Full list of author information is available at the end of the article
} 


\section{Background}

Synthetic polymers are of importance in modern science and technology as they are essential to our daily life with a wide range of applications in various fields such as packaging, agriculture, food, consumer products and medical devices. In the past few decades, considerable attention has been focused on the biodegradable polymers due to their unique biodegradability, enhanced biocompatibility as well as flexible physical and mechanical properties. In particular, biomedical applications of biodegradable polymers generate an enormous amount of interest in research. Biodegradable polymer is a specific type of polymer that breaks down into natural byproducts such as gases $\left(\mathrm{CO}_{2}, \mathrm{~N}_{2}\right)$, water, biomass and inorganic salts after its intended purpose. Applications can be found in drug delivery systems, surgical implants and other medical applications (Gao et al. 2015). Amongst, Poly-lactic acid (PLA) attracted most of the interest. PLA is a semi-crystalline thermoplastic polymer with high strength and modulus, and has been widely used in medical implants in the forms of rods, plates, pins as well as meshes (Pulapura \& Kohn 1992). In addition, PLA can be easily processed into a wide range of products by extrusion, injection moulding, compression moulding as well as film and sheet casting (Lim et al. 2008). PLA can come under the stereo-isomeric forms such as Poly-l-lactic acid (PLLA) and Poly-d-lactide (PDLA), with minor difference in thermal properties (e.g., melting temperature, glass transition temperature and crystallinity).

Significant amount of research has been conducted to study the mechanical properties of biodegradable polymer PLA (Bartkowiak-Jowsa et al. 2011; Liang et al. 2013; Lu et al. 2007; Rezgui et al. 2005; Isabelle \& Lan 2009; Hamad et al. 2015; Liu \& Zhang 2011; Rasal et al. 2010). For instance, Bartkowiak-Jowsa et al. (2011) carried out uniaxial tensile tests to characterize the stressstrain behaviour of several biodegradable polymers, including poly-lactide (PLA), poly-caprolactone (PCL), poly (L-lactide) (PLLA) and poly (lactide-co-glycolide) (PGLA). Their results showed that PLA (PLLA) is a typical brittle material, with relatively high Young's modulus but limited deformation at failure when compared to PCL/PGLA. In fact, mechanical brittleness or poor elongation ( $\leq 3 \%$ ), as well as low strength, of PL(L)A has limited its medical applications considerably. As such, some other polymers (i.e., PCL and PES) have been proposed to fabricate PLA blends for improved mechanical properties. Liang et al. (2013) investigated the tensile mechanical properties of PLLA/PCL/nano- $\mathrm{CaCO}_{3} \mathrm{com}-$ posite and evaluated the effects of blend composition on their mechanical properties. Their results showed that PCL content is helpful to improve the ductility of PLLA composite but with a sacrifice of the strength. Lu et al.
(2007) examined the tensile mechanical properties of PLLA/PES composites by tensile testing and observed that the Young's modulus increased significantly with the increase of weight fraction of PES but with a dramatic decrease of the elongation at break. In addition, the increase of temperature can also affect the ductility of the PLA as shown in Rezgui et al. (2005) who investigated the mechanical behaviour of PLA upon stretching at $50{ }^{\circ} \mathrm{C}$. It was found that PLA underwent extensive plastic deformation, due to a dramatic reduction of yield stress, followed by a progressively increasing strain hardening. It has also been proved that the mechanical properties of PLA are less dependent on the molecular weight. For example, the tensile strength rises $20 \%$ only when the molecular weight increases from 107,000 to $550,000 \mathrm{~g} / \mathrm{mol}$ (Isabelle \& Lan 2009). As reviewed by Hamad et al. (2015), PLA has good mechanical properties, especially tensile Young's modulus and tensile strength, but limited elongation at fracture and low impact strength when compared to other traditional polymers. To improve its ductility, PLA can be blended with polymeric tougheners (e.g., PCL, PBS, PPC and PHA) and the effects can be controlled by varying size, volume fraction, substructure and inherent property of the dispersed toughening phase (Liu \& Zhang 2011). As reported in Rasal et al. (2010), surface- and bulkmodification strategies could be used to toughen PLA, but usually associated with a loss of tensile strength or modulus. They pointed out that the development of PLA-nanocomposites may be a promising approach to improve PLA properties.

Numerical methods have also been widely applied to understand, evaluate and predict the mechanical deformation of biodegradable polymers by means of a variety of material models. For instance, the BergströmBoyce viscoelastic model can be used to simulate the performance of polymers undergoing large deformation (Bergström \& Boyce 1998). The hyper-elastic constitutive relationship, such as the Neo-Hookean and the Mooney-Rivlin models, was used by Vieira et al. (2011) to predict the mechanical deformation of PLA and PCL blend. Vieira et al. (2014) also performed a parametric study based on the adapted Bergström-Boyce model for PLA-PCL blend to simulate three-dimensional timedependent mechanical deformation during hydrolytic degradation. The adapted model was proved to be able to simulate more relevant phenomena such as stress relaxation, creep and inelastic strain measured experimentally. Muliana and Rajagopal (2012) modified a quasilinear viscoelastic (QVL) model to analyse the response of nonlinear viscoelastic biodegradable polymer PLLA by considering the change of material parameters during degradation process. They identified that the stressstrain response was less sensitive to strain rates 


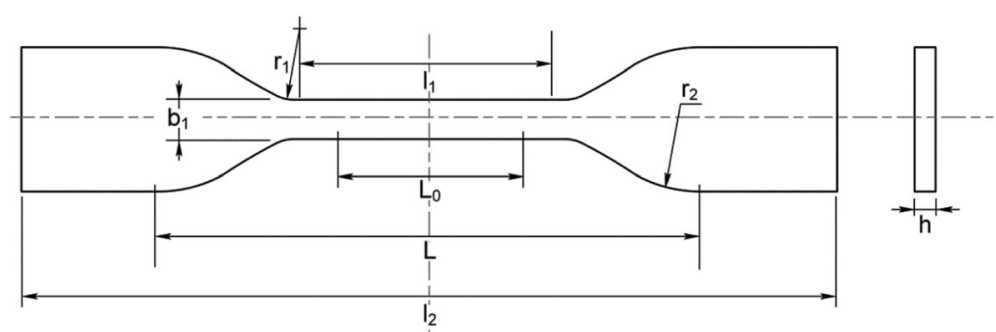

Fig. 1 Sketch for the dog-bone specimen

which agreed with experimental results. So far, modelling of biodegradable polymers has been mostly carried out by considering the elastic/viscoelastic (i.e., time-dependent elastic) deformation and the effects of degradation on the elastic properties of the polymers. There is little research on modelling of biodegradable polymer PLA by considering the viscoplastic deformation, i.e., time-dependent plastic deformation, which is critically important for accurate capture of the material behaviour, especially in modelling of loading-bearing components and devices such as PLLA stents which rely on plastic deformation to function properly.

The present study aims to study the potential in improving the ductility of PLA by blending with PBS in varied weight ratios. Polybutylene Succinate (PBS) is one of the newest biopolymers under development, and belongs to biodegradable aliphatic polyester. It has high crystallinity, a melting point of about $90{ }^{\circ} \mathrm{C}$ $\sim 120^{\circ} \mathrm{C}$ and a glass transition temperature of around $-45 \sim-10{ }^{\circ} \mathrm{C}$ (Foin et al. 2014; Papageorgiou et al. 2007). Moreover, PBS has good material processability, even better than PLA, and can be easily processed at relatively low cost as compared with other biodegradable polymers (Kanemura et al. 2012). Zhao et al. (2005) studied the biodegradation behaviour of PBS under controlled composting conditions, and the degradation process exhibited three phases. The

Table 1 Dimensions for PLA/PBS dog-bone specimen (corresponding to Fig. 1)

\begin{tabular}{lll}
\hline Symbol & Explanation & Value $(\mathrm{mm})$ \\
\hline $\mathbf{I}_{2}$ & Overall length, minimum & 75 \\
$\mathbf{I}_{1}$ & Length of narrow parallel-sided portion & 25 \\
$\mathbf{b}_{1}$ & Width of narrow parallel-sided portion & 4 \\
$\mathbf{r}_{1}$ & Small radius & 8 \\
$\mathbf{r}_{2}$ & Large radius & 12.5 \\
$\mathbf{L}$ & Initial distance between grips & 50 \\
$\mathbf{L}_{0}$ & Gauge Length & 20 \\
$\mathbf{h}$ & Thickness & 1 \\
\hline
\end{tabular}

biodegradation in the first phase was slow, got accelerated in the second phase, and showed a leveling-off in the third phase. Chieng et al. (2010) reported that the strength and modulus of PBS can be improved effectively by blending with poly(butylene adipate-coterephthalate) (PBAT) and organo-modified montmorillonite (OMMT). Similarly, Okamoto et al. (2003) prepared PBS/layered-silicate nanocomposites by melting extrusion, and results showed that there was significant improvement in tensile modulus but with a compromise of tensile yield strength.

In this paper, PLA/PBS blends, with weight ratios at 90/10, 70/30, 50/50, 30/70 and 10/90, were produced by roller mixing at melting condition. Thermal properties were characterized by Differential Scanning Calorimetry (DSC) for the range of PLA/PBS blends produced. The crystalline morphology of the PLA/PBS blends was studied by Optical Microscopy. Mechanical properties were studied by uniaxial tensile testing. Also, the established Chaboche model was applied to simulate the viscoplastic deformation of PLA/PBS blends, with calibration against the experimental results.

\section{Methods \\ Materials}

Both poly (lactic-acid) (PLA) (Ingeo PLA 4043D, NatureWorks LLC) and poly (butylene succinate) (PBS) (RJ503S, Hangzhou Ruijiang Chemical Co. Ltd.) were purchased for this study. PLA 4043D is a type of biopolymer, and its melting temperature is in the range of $145-160{ }^{\circ} \mathrm{C}$. The density is $1.24 \mathrm{~g} / \mathrm{cm}^{3}$, and tensile strength is about $110 \mathrm{MPa}$. PBS RJ503S has a melting point in the range of $110-116{ }^{\circ} \mathrm{C}$ and a density of $1.20-1.28 \mathrm{~g} / \mathrm{cm}^{3}$. The tensile strength is relatively low (35-45 MPa) but the elongation at break can be as high as $300 \%$. Both polymers were provided in the form of pellets.

PLA 4043D is not for medical purpose, but its tensile strength and elongation at break are considered to be very close to that of PLA polymer in medical grade. Considering that polymer PLA in medical grade is far too expensive, polymer PLA 4043D was used as an alternative in this research. Polymer PBS (RJ503S) was 


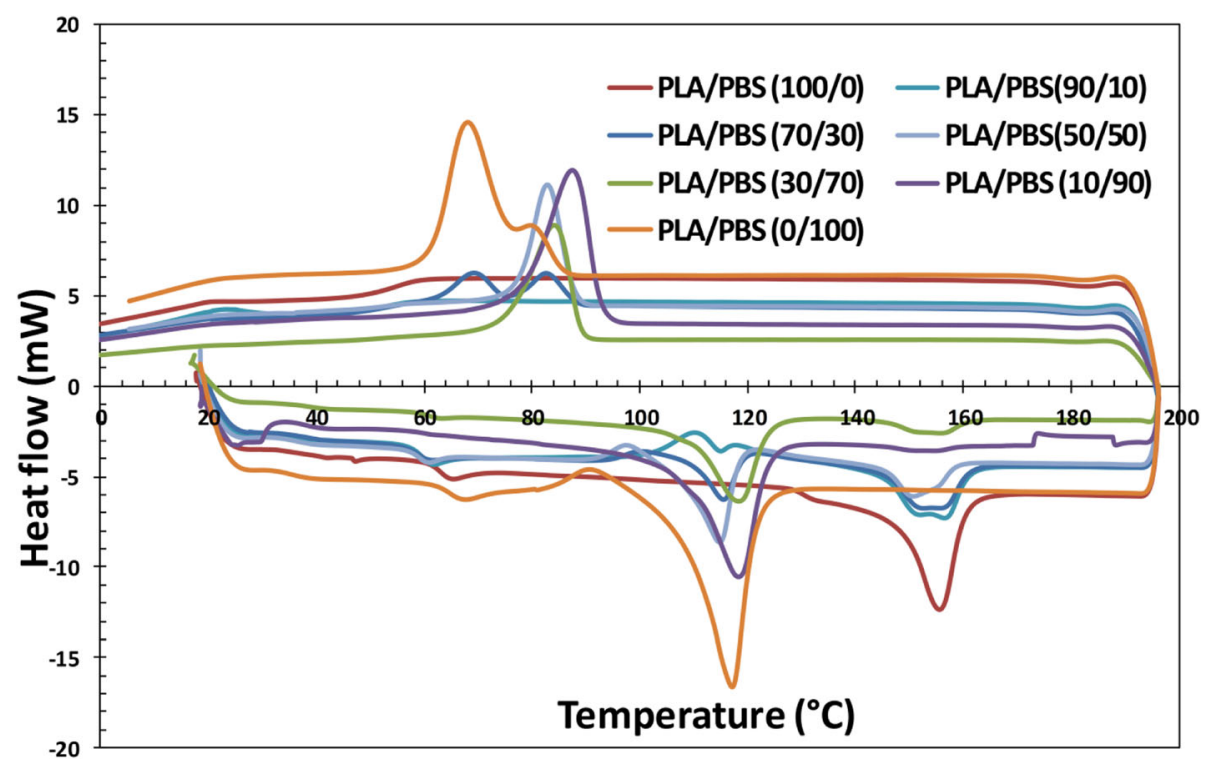

Fig. 2 DSC thermal diagrams of PLA, PBS and their blends including both endotherm and exotherm

chosen in the research because it is very ductile (elongation at break $\geq 300 \%$ ) and used to overcome the brittleness of polymer PLA through blending. However, the tensile strength of PBS is limited (35-45 MPa), so the weight ratio in the blend should be controlled in order to achieve required mechanical strength.
Preparation of PLA/PBS blends and specimens

To prepare PLA/PBS blends, PLA and PBS pellets were pre-dried in the oven at the temperature of $80{ }^{\circ} \mathrm{C}$ for at least $12 \mathrm{~h}$. They were then melted and mixed in HAAKE Polylab OS mixer for $10 \mathrm{~min}$ at a mixing speed of $60 \mathrm{rpm}$ and a temperature of $195{ }^{\circ} \mathrm{C}$. PLA/PBS weight

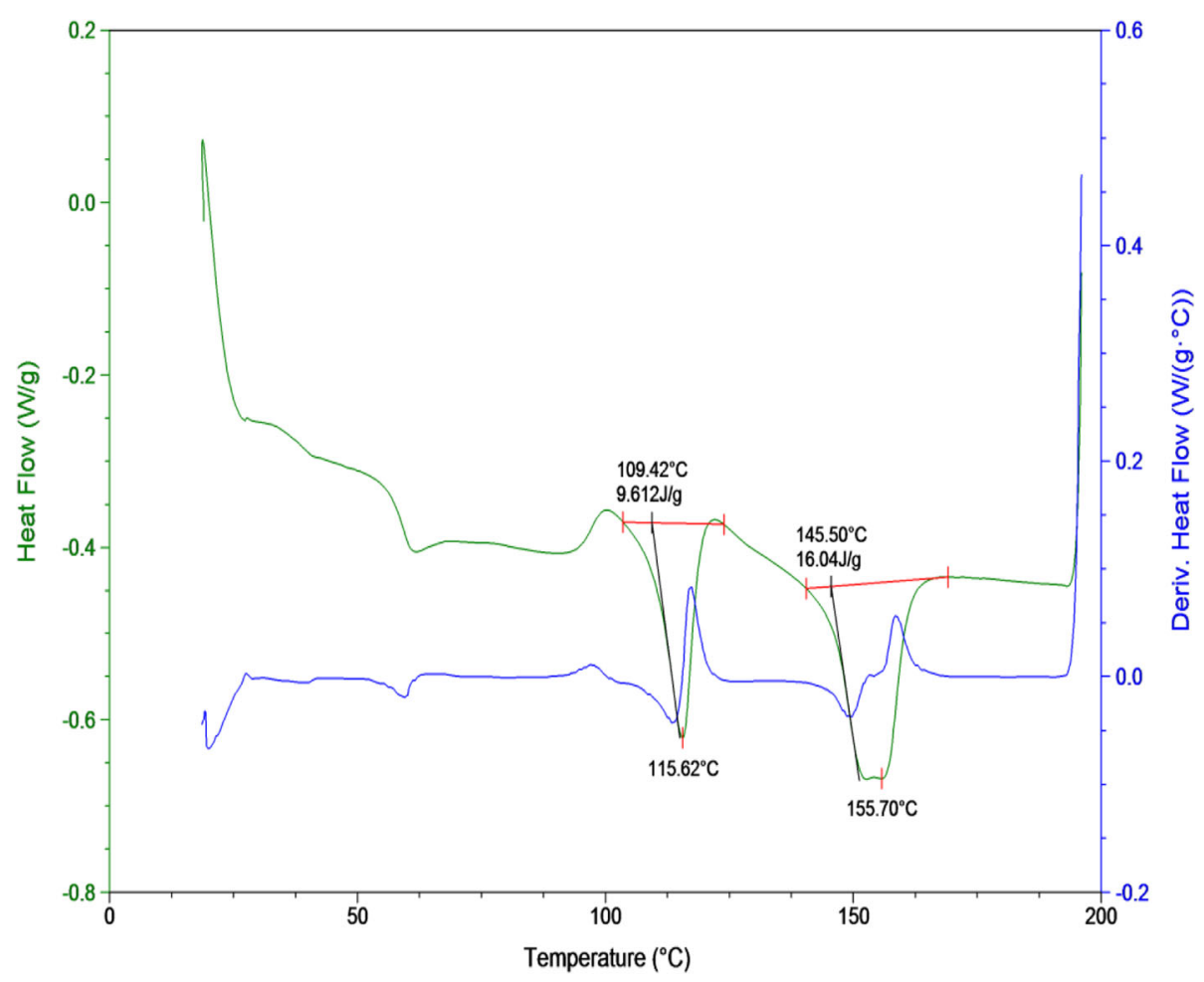

Fig. 3 An example of melting temperature and heat flow calculation for PLA/PBS blend (70/30) 


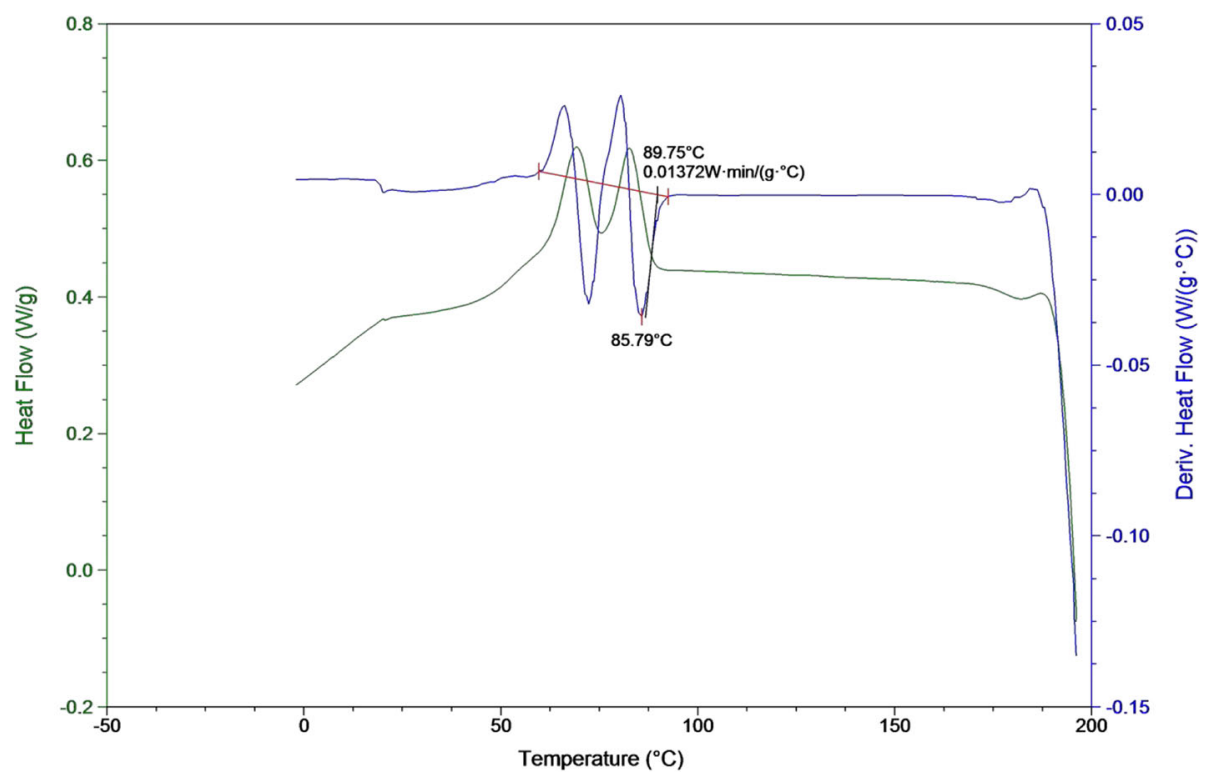

Fig. 4 An example of crystallization temperature and heat flow calculation for PLA/PBS blend (70/30)

ratios of $90 / 10,70 / 30,50 / 50,30 / 70$ and $10 / 90$ were considered.

To produce PLA/PBS specimens, PLA/PBS plates $\left(80 \times 80 \times 1 \mathrm{~mm}^{3}\right)$ were firstly obtained from PLA/PBS blends by compression moulding method. The temperature for compression moulding depended on the melting points of both materials. Those plates were further cut into dog-bone specimens. Pure PLA and PBS specimens were also prepared as control. These dogbone specimens were used for mechanical testing to investigate mechanical properties. The dimensions for the PLA/PBS dog-bone specimens followed the testing Standard ISO-527-2-5A (see Fig. 1 and Table 1).

\section{Characterization of PLA/PBS blends}

\section{Differential scanning calorimetric (DSC)}

TA instruments DSC 2010 differential scanning calorimeter was used to evaluate the thermal behaviour of PLA/ PBS blends. Non-isothermal run was performed for PLA, PBS and their blends. The materials were heated to $200{ }^{\circ} \mathrm{C}$ at a rate of $10{ }^{\circ} \mathrm{C} / \mathrm{min}$, cooled down to room

Table 2 Thermal properties of PLA in the blends

\begin{tabular}{llll}
\hline PLA/PBS blend & $\begin{array}{l}T_{m^{\rho L A}} \\
{ }^{\rho}\end{array}$ & $\begin{array}{l}\Delta H_{m^{\rho L A}} \\
\mathrm{~J} / \mathrm{g}\end{array}$ & $\begin{array}{l}X_{c^{\rho L A}} \\
\%\end{array}$ \\
\hline $100 / 0$ & 148 & 18.00 & 19.35 \\
$90 / 10$ & 145 & 20.00 & 23.89 \\
$70 / 30$ & 145 & 16.04 & 24.64 \\
$50 / 50$ & 146 & 9.38 & 20.17 \\
$30 / 70$ & 147 & 3.60 & 12.90 \\
$10 / 90$ & 145 & 1.49 & 16.02 \\
\hline
\end{tabular}

temperature at the same rate, and then reheated to $200^{\circ}$ C in DSC system. Crystallisation temperature, degree of crystallinity and melting temperature of the blends were identified from the DSC curves. The degree of crystallinity was calculated by the following equation:

$$
\begin{aligned}
& X_{c^{P L A}}(\%)=\left[\frac{\Delta H_{m^{P L A}}-\Delta H_{c^{P L A}}}{\Delta H_{m^{P L A, 0}} \times W^{P L A}}\right] * 100 \\
& X_{c^{P B S}}(\%)=\left[\frac{\Delta H_{m^{P B S}}}{\Delta H_{m^{P B S, 0}} \times W^{P B S}}\right] * 100
\end{aligned}
$$

where $W^{P L A}$ and $W^{P B S}$ is the PLA and PBS weight fraction in the blend system, $\Delta \mathrm{H}_{\mathrm{c}^{\mathrm{PLA}}}$ stands for the enthalpy of crystallization for PLA in the exothermal process, and $\Delta H_{m}^{0}$ is the heat of fusion for $100 \%$ polymer crystal $\Delta$ $H_{m^{P L A, 0}}=93.0 \mathrm{~J} / \mathrm{g}$ and $\left.\Delta H_{m^{P B S, 0}}=210.0 \mathrm{~J} / \mathrm{g}\right)$.

\section{Optical microscopy analysis}

Leica DMRX microscope was used to examine the crystalline structure of the pure PLA, PBS and their blends.

Table 3 Thermal properties of PBS in the blends

\begin{tabular}{lllll}
\hline PLA/PBS blend & $\begin{array}{l}T_{C^{P B S}} \\
{ }^{0} \mathrm{C}\end{array}$ & $\begin{array}{l}T_{m^{P B S}} \\
{ }^{P}\end{array}$ & $\begin{array}{l}\Delta H_{m^{P B S}} \\
\mathrm{~J} / g\end{array}$ & $\begin{array}{l}X_{C^{P B S}} \\
\%\end{array}$ \\
\hline $100 / 0$ & - & - & - & - \\
$90 / 10$ & - & - & - & - \\
$70 / 30$ & 90 & 110 & 9.61 & 15.25 \\
$50 / 50$ & 88 & 106 & 27.64 & 26.32 \\
$30 / 70$ & 89 & 109 & 26.86 & 18.27 \\
$10 / 90$ & 93 & 108 & 43.61 & 23.07 \\
$0 / 100$ & 77 & 108 & 68.59 & 32.66 \\
\hline
\end{tabular}



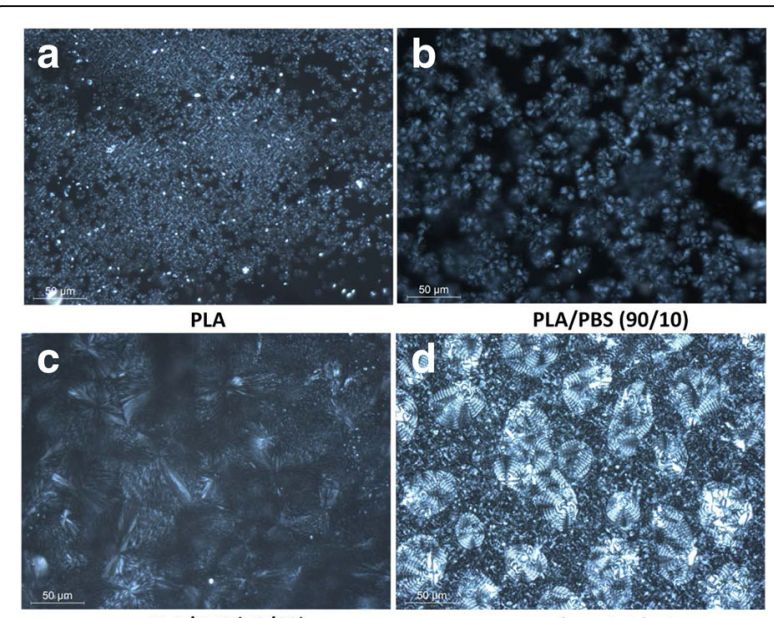

PLA/PBS (70/30)

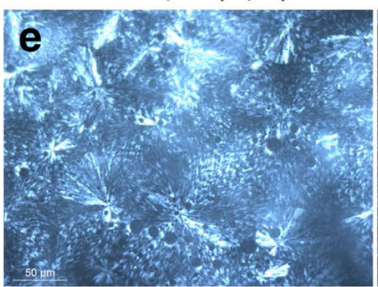

PLA/PBS (30/70)

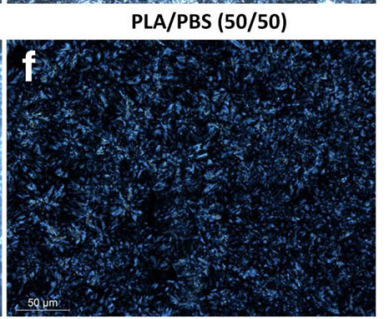

PBS

Fig. 5 Optical microscopy images for PLA/PBS blends: a PLA; b PLA/ PBS (90/10); c PLA/PBS (70/30), d PLA/PBS (50/50); e PLA/PBS (30/70); f $\mathrm{PBS}$

Material samples were heated to melt fully into very thin film on a hot stage at the temperature of $200{ }^{\circ} \mathrm{C}$, and then squeezed between cover glasses. They were then put into the oven at a temperature of $80{ }^{\circ} \mathrm{C}$ for $30-\mathrm{min}$ crystallization, and finally quenched to room temperature $\left(20{ }^{\circ} \mathrm{C}\right)$.

\section{Mechanical testing}

Mechanical properties were evaluated by uniaxial tensile testing on dog-bone samples at room temperature. Instron machine (Instron 2716-010, maximum load $50 \mathrm{kN}$ ) was used for the experiments with a loading rate of $10 \mathrm{~N} / \mathrm{min}$. Stress-strain behaviour of the blends was obtained to extract the elastic modulus, tensile strength and elongation at break. In particular, to study the viscous behaviour, strain-controlled tests were also carried out for the blended specimens under two strain rates, i.e., $0.01 / \mathrm{s}$ and $0.0001 / \mathrm{s}$. It should be pointed out that the strain rate was specified for each test and the machine automatically kept this rate constant during the whole process. In fact, the displacement against time was monitored for all specimens and showed a straight line, confirming the steady-state or constant strain rate during the test. Stress-strain responses were recorded and also simulated by using the visco-plasticity model described below.

\section{Numerical model}

The numerical model is basically the visco-plastic constitutive equations established by Chaboche, with both isotropic and kinematic hardening variables. This is a well-established constitutive model mostly used to describe the viscoplastic behaviour of metallic materials, and was applied here, for the first time we believe, for polymers. Essentially, for uniaxial and small deformation, the strain rate $\dot{\varepsilon}$ is additively decomposed into an elastic component $\dot{\varepsilon}_{e}$ and an inelastic component $\dot{\varepsilon}_{p}$ (Chaboche 2008):

$$
\dot{\varepsilon}=\dot{\varepsilon}_{e}+\dot{\varepsilon}_{p}
$$

The stress $\sigma$ can be obtained from the elastic strain $\dot{\varepsilon}_{e}$ by using Hook's law:

$$
\dot{\sigma}=E \dot{\varepsilon}_{e}
$$

where $E$ is the Young's modulus of the material. The inelastic strain $\varepsilon_{p}$ represents both plastic and creep strains, and can be expressed by a power law relationship:

$$
\dot{\varepsilon}_{p}=\left\langle\frac{f}{Z}\right\rangle^{n} \frac{\partial f}{\partial \sigma}
$$

where $f$ is the Von Mises yield function, $Z$ and $n$ are viscous constants and the bracket is defined by:

$$
\langle x\rangle=\left\{\begin{array}{l}
x, x \geq 0 \\
0, x<0 .
\end{array}\right.
$$

Based on the von Mises yield criterion, the yield function $\mathrm{f}$ for uniaxial loading situation is defined as:

$$
f(\sigma, \alpha, R, k)=|\sigma-\alpha|-R-k \leq 0,
$$

where $\alpha$ is the non-linear kinematic hardening variable, $R$ is the isotropic hardening variable and $k$ is the initial value of the yield stress. Plastic flow occurs under the condition $f=0$ and $\frac{\partial f}{\partial \sigma}: \dot{\sigma}>0$. For this viscoplastic model, the yield surface is able to move and expand but the stress in excess of the yielding stress is now permitted and often called as "overstress".

According to Chaboche, the kinematic $(\alpha)$ and isotropic (R) hardening variables evolve through the following rules (Chaboche 1989):

$$
\dot{\alpha}=C\left(a \dot{\varepsilon}_{p}-\alpha \dot{p}\right) \text { and } \dot{R}=b(Q-R) \dot{p}
$$

where $C, a, b$ and $Q$ are material constants which determine the strain hardening behaviour of the material under monotonic and cyclic loading. Basically, $a$ is the saturated value of the kinematic hardening variable, and $C$ indicates the speed with which the saturation is reached. While $Q$ is the asymptotic value of the isotropic variable $R$ at saturation and $b$ indicates the speed 


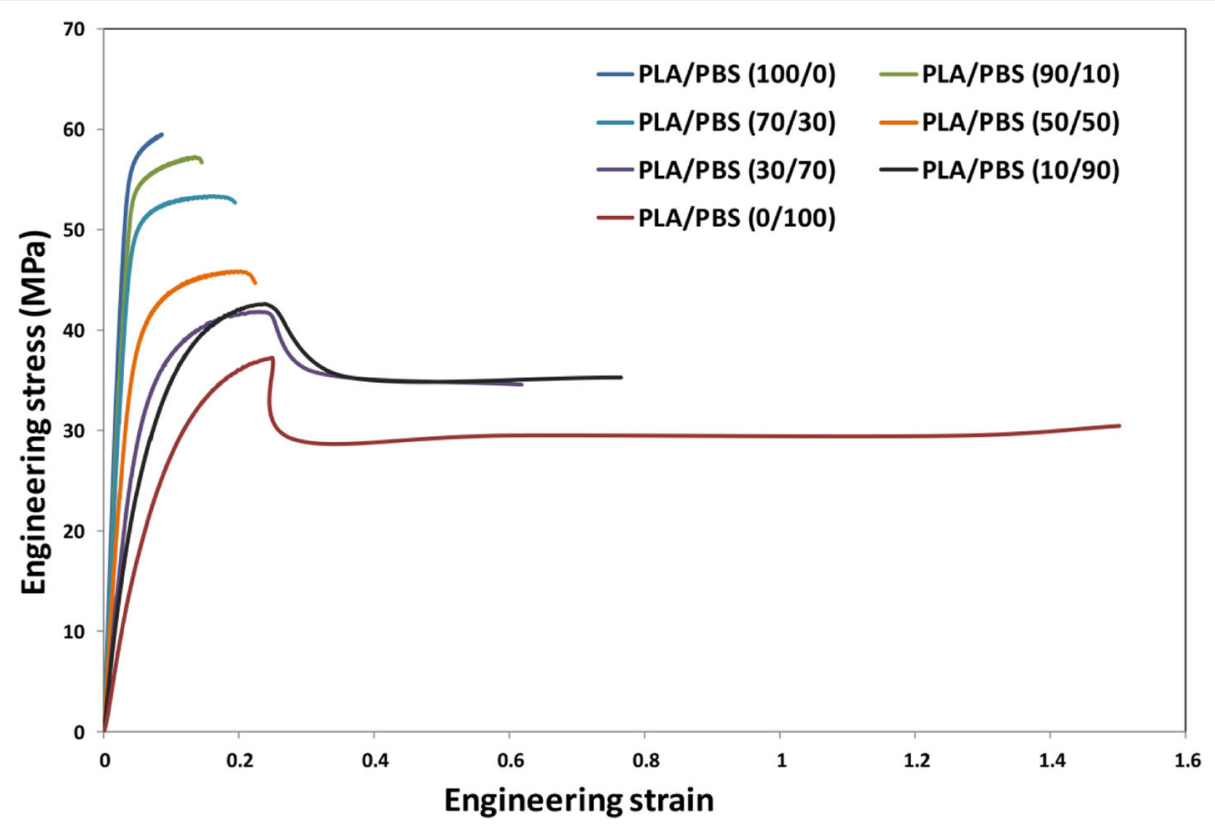

Fig. 6 Uniaxial tensile stress-strain curves for PLA/PBS blends tested at a loading rate of $10 \mathrm{~N} / \mathrm{min}$

towards the saturation. In the above equation, $\dot{p}$ is defined as the accumulated inelastic strain rate given by:

$$
\dot{p}=\left\langle\frac{f}{Z}\right\rangle^{n}
$$

The above viscoplastic constitutive equations contain several material parameters such as $E, k, b, Q, C, a, Z$ and $n$, which are to be determined from the experimental data.

\section{Results and discussion DSC analysis}

Generally, DSC analysis is one of reliable method for measurement of melting temperature and crystallization of semi-crystalline polymer. Figure 2 shows DSC diagrams for pure PLA, pure PBS and their blends. In terms of endothermal process, it can be noticed that there is only one peak occurred in the endothermal process for either pure PLA or PBS sample, which is at about 148 and $108{ }^{\circ} \mathrm{C}$, respectively. All other blends exhibit two melting peaks in the endothermal process, which are around $100 \sim 120$ and $140 \sim 160{ }^{\circ} \mathrm{C}$, respectively. An example is given in Fig. 3 to indicate the method for calculation of melting temperature and heat flow for PLA/PBS $(70 / 30)$ blend. It can be concluded from these thermal diagrams that polymer PLA and PBS are immiscible by mechanical mixing method.

While in the exothermal process, pure PLA and PLA/PBS (90/10) blend have definitely slow crystallization process, which can hardly be captured in the DSC thermal diagrams. Figure 4 gives an example for the calculation of crystallization temperature and heat flow for PLA/PBS (70/30) blend. The crystallization temperature of pure PBS is calculated as $77{ }^{\circ} \mathrm{C}$. In particular, there are two exothermal peaks in the PLA/PBS $(70 / 30)$ blend. Therefore, it might be considered that PBS composition would improve PLA crystallization behaviour. Thermal properties of pure polymer and their blends are summarized in Tables 2 and 3. It is clear that the crystallinity of PLA in the blend increased from 19.35 to $24.64 \%$ with the addition of $30 \%$ PBS, and then slightly decreased with the further increase of PBS content. On the other hand, as shown in Table 3 , the crystallinity of PBS was compromised by the blending with PLA.

The crystallization temperature $\left(T_{c^{P L A}}\right)$ and enthalpy $\left(\Delta H_{c^{P L A}}\right)$ for PLA composition are missing in Table 2 . This is because the crystallization behaviour was not found for PLA in the exothermal process. Table 3 gives the thermal properties for PBS composition in PLA/PBA blend. For blend PLA/PBS $(100 / 0)$, it is pure PLA without PBS, so it is empty in the first column. Also for PLA/PBS $(90 / 10)$ blend, a peak in the exothermal process could not be identified from the DSC diagrams, so the relevant values are also missing. It might be because there is very limited PBS composition in this blend, and it is difficult to be characterized in the DSC testing. 


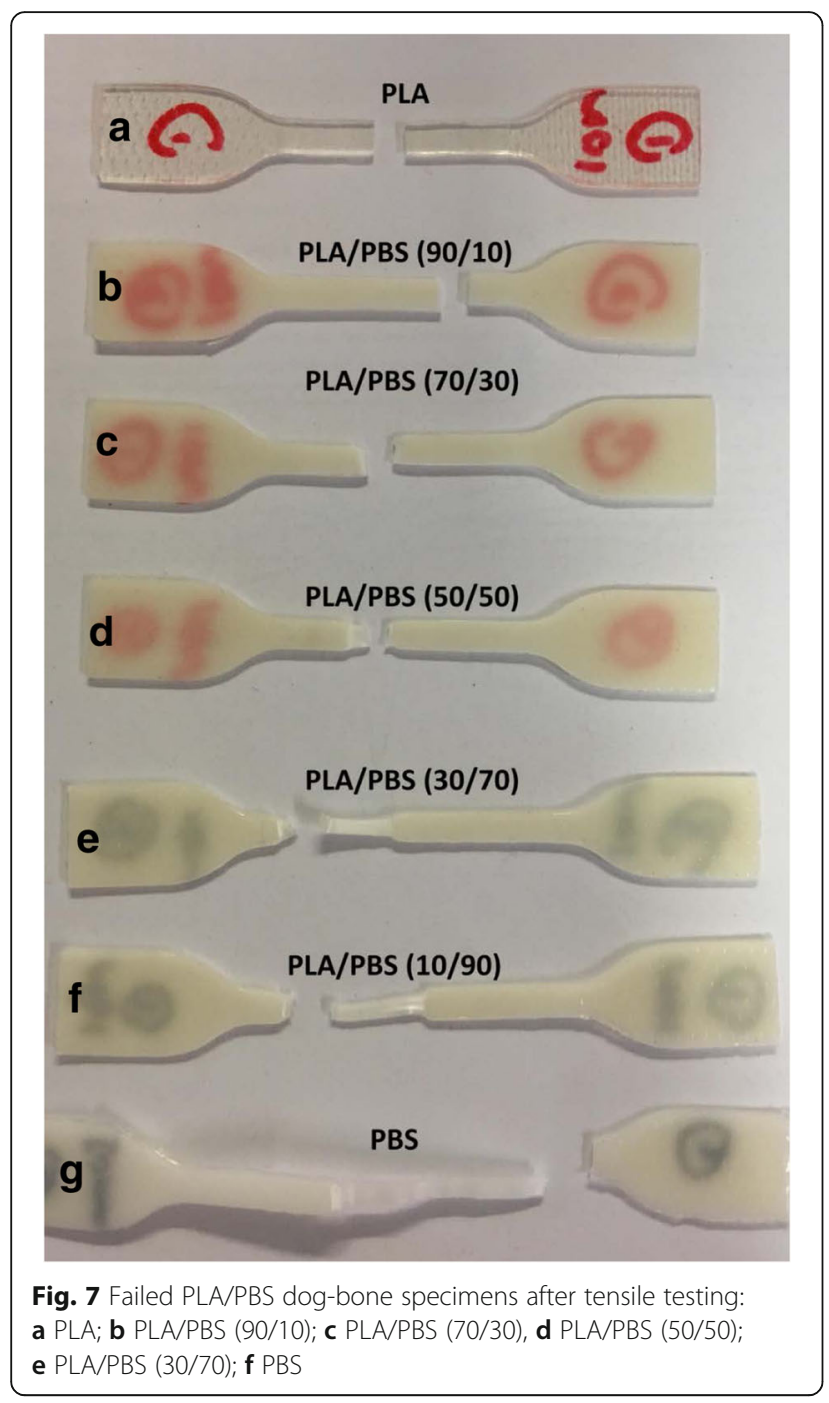

\section{Optical microscopy analysis}

In order to characterize what happened to the PLA/PBS blend system, optical microscopy analysis was conducted to observe the crystalline morphology and the results are shown in Fig. 5. It is clear that the crystal of pure polymer PLA is small in size and quite uniformly dispersed in terms of distribution (see Fig. 5a). The PBS crystal is also small in size but with a rather nonuniform distribution (see Fig. 5f). Moreover, the crystal in the PLA/PBS blends tends to increase in size with the addition of PBS content. In particular, it clearly shows that the crystal in PLA/PBS (70/30) blend tends to have a bigger size and good spreading. It could be considered that the addition of PBS content aids with the crystallization process of polymer PLA, leading to altered mechanical properties (Yokohara \& Yamaguchi 2008; Stoyanova et al. 2014).

\section{Mechanical properties of PLA/PBS blends}

Figure 6 shows the stress-strain curves of the PLA/PBS blends with various weight ratios. It should be noted that three specimens were tested for each blend, and the mean values were used to produce this stress-strain diagram. Pure PBS and blends with more than $50 \%$ of PBS such as PLA/PBS (10/90) and PLA/PBS (30/70) showed distinctive ductile behaviour, with the occurrence of necking behaviour (Fig. 7). While pure PLA and blends with less than $50 \%$ of PBS such as PLA/PBS (90/10) and PLA/PBS (70/30) showed the nature of brittle fracture, with no sign of necking phenomenon.

Furthermore, tensile modulus, elongation and strength were obtained from the stress-strain curves, and presented in Figs. 8, 9 and 10, with a normalisation to those of pure PLA. As shown in Fig. 8, pure PLA polymer shows

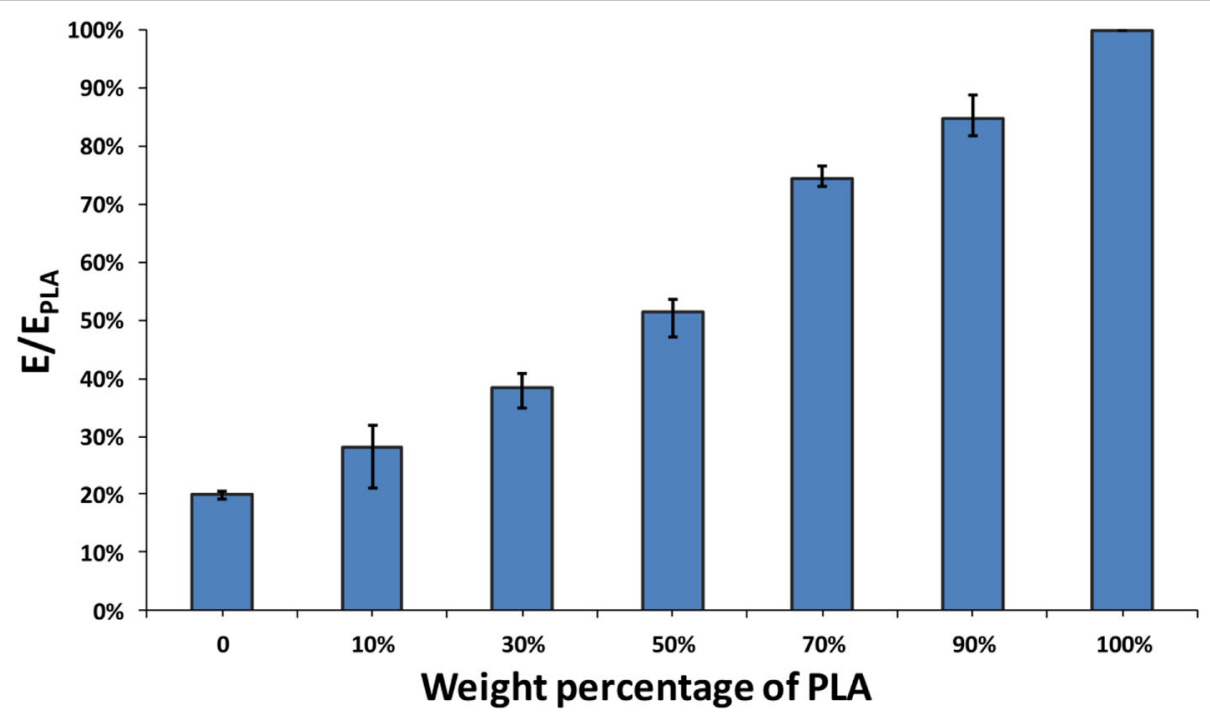

Fig. 8 Tensile modulus for PLA/PBS blends as a function of PLA weight percentage, with normalisation against that of pure PLA 


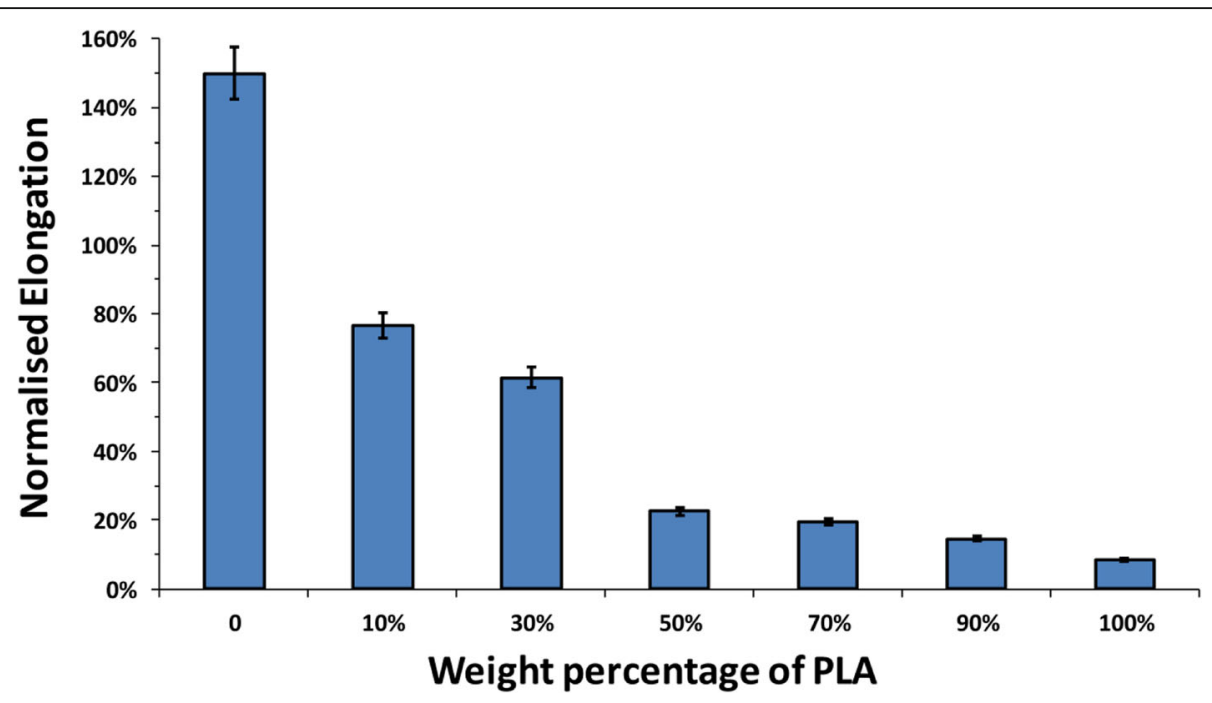

Fig. 9 Elongation for PLA/PBS blends as a function of PLA weight percentage, with normalisation against that of pure PLA

the highest tensile modulus (around 1.8 GPa), and the tensile modulus of PLA/PBS (90/10, 70/30) blends were reduced to 1.6 and 1.4 GPa, respectively. Further decrease in modulus was obtained with the addition of more PBS. These results are similar to the results obtained from PLA/PBS blends prepared by either melt spinning technique (Jompang et al. 2013) or Haake RHeomix OS mixer (Deng \& Thomas 2015). The elongation of pure PLA is really limited $(8 \%)$ and further improved by the blending with ductile PBS polymer, as shown in Fig. 9. Specifically, the elongation increased to 12.5 and $15.4 \%$ for the addition of 10 and $30 \%$ PBS, respectively. The PBS/PLA blends with weight ratio 70/30 and 90/10 are dominated by $\mathrm{PBS}$, and consequently they showed significant elongation under tension. Tensile strength of PLA/PBS blends exhibits the same trend as the tensile modulus (see Fig. 10). All results reveal that the brittleness of pure PLA can be improved by blending with ductile PBS using mechanical mixing technique, but with a compromise of tensile modulus and strength (Bhatia et al. 2007).

\section{Strain rate effect and model simulation}

Tensile stress-strain curves (plotted in symbols) are shown in Fig. 11 at two different strain rates, i.e., $0.0001 / \mathrm{s}$ and $0.01 / \mathrm{s}$. It is clear that the strain rate has an effect on the stress-strain behaviour of PLA/PBS blends, demonstrating the time-dependent deformation nature of the material, i.e., an increase of stress with the

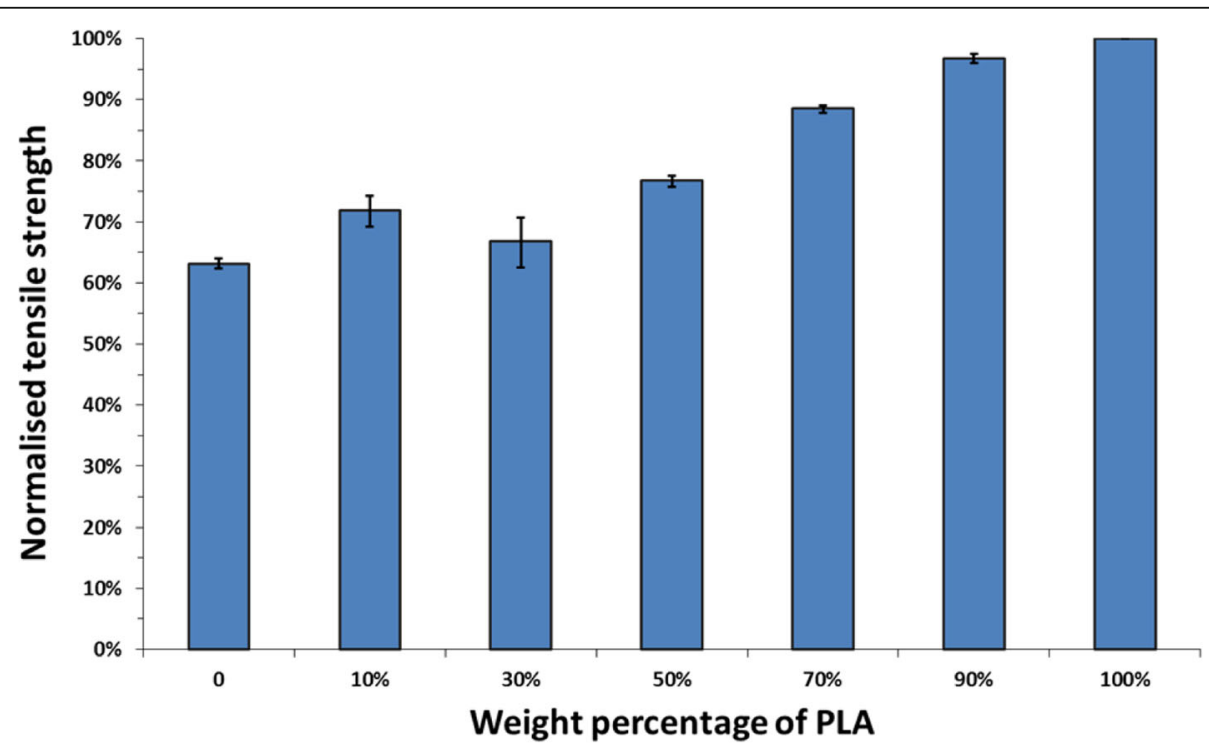

Fig. 10 Tensile strength for PLA/PBS blends as a function of PLA weight percentage, with normalisation against that of pure PLA 


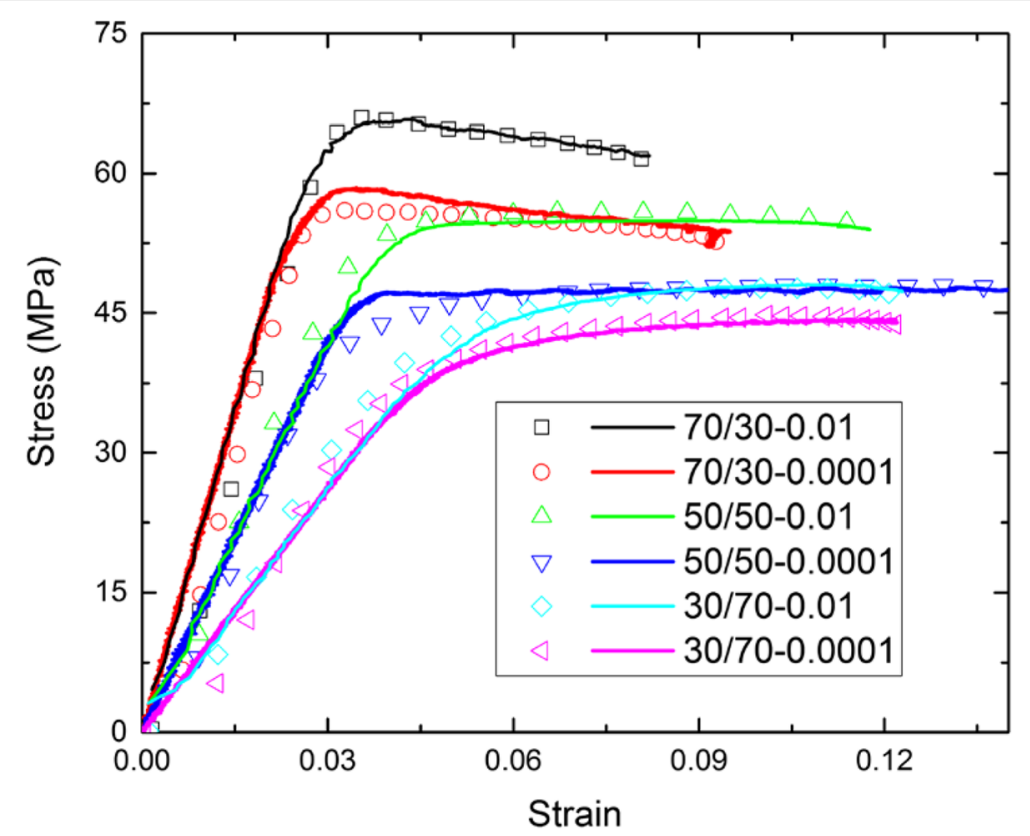

Fig. 11 The rate dependency of stress-strain behaviour for PLA/PBS blends, comparison of experimental and simulation results

increase of strain rate. This time-dependent or viscous behaviour needs to be considered properly in the design and manufacturing of components and devices out of PLA/PBS, including computational modelling.

To model the rate-dependent deformation using the Chaboche model, a simultaneous determination approach was used to obtain the corresponding model parameters for PLA/PBS blends from the test data. For this purpose, an objective function was defined to measure the difference between the experimental stress values and the corresponding simulated stress values for a given set of parameters. The goal is to determine the set of parameters which minimizes the objective function, i.e., the difference between experimental and simulated results. The gradient-based Levenberg-Marquardt algorithm (Dennis

Table 4 Optimised parameter values for the viscoplastic constitutive model

\begin{tabular}{lrrr}
\hline Parameters & \multicolumn{1}{c}{$70 / 30$} & \multicolumn{1}{c}{$50 / 50$} & $30 / 70$ \\
\hline E (MPa) & 2296.78 & 1395.73 & 870.15 \\
$Z$ & 33.19 & 31.49 & 17.66 \\
$\mathrm{~N}$ & 8.06 & 6.92 & 65.59 \\
$\mathrm{C}_{1}$ & 413.82 & 610.23 & 590.21 \\
$\mathrm{a}_{1}(\mathrm{MPa})$ & 9.59 & 5.16 & 4.87 \\
$\mathrm{C}_{2}$ & 51.73 & 104.67 & 85.34 \\
$\mathrm{a}_{2}(\mathrm{MPa})$ & 5.67 & 4.27 & 5.22 \\
$\mathrm{~b}$ & 36.08 & 147.51 & 3.45 \\
$\mathrm{Q}(\mathrm{MPa})$ & -11.48 & -5.23 & 8.35 \\
$\mathrm{k}(\mathrm{MPa})$ & 40.12 & 35.16 & 29.77 \\
\hline
\end{tabular}

Jr et al. 1996) has been used to determine the optimum set of material parameters in an iterative manner. The algorithm is essentially a nonlinear least square method. In order to achieve the global minimum of the objective function, different sets of initial parameters were adopted to find a stabilized solution. Then, a perturbation was given to the stabilized solution and the LevenbergMarquardt procedure was re-started to check if the optimization algorithm returns a better solution or the same one. Based on the optimisation described above, the calibrated model parameters are listed in Table 4. Note that the Young's modulus E was derived directly from the linear part of the tensile stress-strain curve. Also the values of the parameters given in Table 4 need to be treated as a group and any individual value may be meaningless, since the parameter values were optimised together in this work. The simulated results (in lines) together with the experimental results are plotted in Fig. 11. Good correlation between the simulation and the experimental data was obtained for the two strain rates considered. This is very useful for more appropriate modelling-based assessment of mechanical performance of components, devices and structures made out of polymers.

\section{Conclusions}

PLA/PBS blends were obtained by mechanical mixing technique, and dog-bone specimens were manufactured by compression moulding. The crystallinity of PLA/PBS blends increased with the addition of PBS content. The mechanical properties of PLA/PBS blends were studied 
by conducting tensile testing, and results showed that the brittleness of pure PLA can be improved by blending with ductile PBS using mechanical mixing technique, but with a loss of stiffness and strength. The tensile tests at different strain rates confirmed the time-dependent plastic deformation nature of the blends, i.e., viscoplasticity. The study also showed that the time-dependent material behaviour can be simulated by the Chaboche viscoplastic model with nonlinear hardening variables.

\section{Authors' contributions}

TQ carried out the technical work; LGZ and MS conceived and supervised the work. All authors contributed to the writing and editing of the paper. All authors read and approved the final manuscript.

\section{Competing interests}

The authors declare that they have no competing interests.

\section{Author details}

'Wolfson School of Mechanical, Electrical and Manufacturing Engineering, Loughborough University, Loughborough LE11 3TU, UK. ${ }^{2}$ Department of Materials, Loughborough University, Loughborough LE11 3TU, UK.

Received: 14 September 2016 Accepted: 3 November 2016

Published online: 16 November 2016

\section{References}

Gao X, Shi Z, Liu C, Guang Y, Sevostianov I, Silberschmidt W (2015) Inelastic behaviour of bacterial cellulose hydrogel: in aqua cyclic tests. Polym Test 44: 82-92

Pulapura S, Kohn J (1992) Trends in the development of bioresorbable polymers for medical applications. J Biomater Appl 6:216-250

Lim LT, Auras R, Rubino M (2008) Processing technologies for poly (lactic acid),". Prog Polym Sci 33:820-852

Bartkowiak-Jowsa M, Będziński R, Chłopek J, Filipiak J, Szaraniec B (2011) Comparative analysis of the deformation characteristics of biodegradable polymers considered as a material for vascular stents. Polimery-W 56:224-231

Liang JZ, Duan DR, Tang CY, Tsui CP, Chen DZ (2013) Tensile properties of PLLA/ $\mathrm{PCL}$ composites filled with nanometer calcium carbonate. Polym Test 32: 617-621

Lu J, Qiu Z, Yang W (2007) Fully biodegradable blends of poly (I-lactide) and poly (ethylene succinate): miscibility, crystallization, and mechanical properties. Polymer 48:4196-4204

Rezgui F, Swistek M, Hiver JM, G'sell C, Sadoun T (2005) Deformation and damage upon stretching of degradable polymers (PLA and PCL). Polymer 46: 7370-7385

Isabelle V, Lan T (2009) Review: biodegradable polymers. Materials 2:307-344

Hamad K, Kaseem M, Yang HW, Deri F, Ko YG (2015) Properties and medical applications of polylactic acid: a review. Express Polym Lett 9:435-455

Liu H, Zhang J (2011) Research progress in toughening modification of poly (lactic acid). J Polym Sci B Polym Phys 49:1051-1083

Rasal RM, Janorkar AV, Hirt DE (2010) Poly (lactic acid) modifications. Prog Polym Sci 35:338-356

Bergström JS, Boyce MC (1998) Constitutive modeling of the large strain timedependent behavior of elastomers. J Mech Phys Solids 46:931-954

Vieira AC, Marques AT, Guedes RM, Tita V (2011) Material model proposal for biodegradable materials. Procedia Eng 10:1597-1602

Vieira AC, Guedes RM, Tita V (2014) Constitutive modeling of biodegradable polymers: hydrolytic degradation and time-dependent behavior. Int J Solids Struct 51:1164-1174

Muliana A, Rajagopal KR (2012) Modelling the response of nonlinear viscoelastic biodegradable polymeric stents. Int I Solids Struct 49:989-1000

Foin N, Lee RD, Torii R, Guitierrez-Chico JL, Mattesini A, Nijjer S, Wong P (2014) Impact of stent strut design in metallic stents and biodegradable scaffolds. Int J Cardiol 177:800-808

Papageorgiou GZ, Achilias DS, Bikiaris DN (2007) Crystallization kinetics of biodegradable poly (butylene succinate) under isothermal and nonisothermal conditions. Macromol Chem Phys 208:1250-1264
Kanemura C, Nakashima S, Hotta A (2012) Mechanical properties and chemical structures of biodegradable poly (butylene-succinate) for material reprocessing. Polym Degrad Stab 97:972-980

Zhao JH, Wang XQ, Zeng J, Yang G, Shi FH, Yan Q (2005) Biodegradation of poly (butylene succinate) in compost. J Appl Polym Sci 97:2273-2278

Chieng BW, Ibrahim NA, Wan Yunus WMZ (2010) Effect of organo-modified montmorillonite on poly (butylene succinate)/poly (butylene adipate-coterephthalate) nanocomposites. Express Polym Lett 4:404-414

Okamoto K, Sinha Ray S, Okamoto M (2003) New poly (butylene succinate)/ layered silicate nanocomposites. II. Effect of organically modified layered silicates on structure, properties, meltrheology, and biodegradability. J Polym Sci B Polym Phys 41:3160-3172

Chaboche JL (2008) A review of some plasticity and viscoplasticity constitutive theories. Int J Plasticity 24:1642-1693

Chaboche JL (1989) Constitutive equations for cyclic plasticity and cyclic viscoplasticity. Int J Plasticity 5:247-302

Yokohara T, Yamaguchi M (2008) Structure and properties for biomass-based polyester blends of PLA and PBS. Eur Polym J 44:677-685

Stoyanova N, Paneva D, Mincheva R, Toncheva A, Manolova N, Dubois P, Rashkov I (2014) Poly (I-lactide) and poly (butylene succinate) immiscible blends: from electrospinning to biologically active materials. Mater Sci Eng C 41:119-126

Jompang L, Thumsorn S, On JW, Surin P, Apawet C, Chaichalermwong T, Srisawat N (2013) Poly (lactic acid) and poly (butylene succinate) blend fibers prepared by melt spinning technique. Energy Procedia 34:493-499

Deng Y, Thomas NL (2015) Blending poly (butylene succinate) with poly (lactic acid): ductility and phase inversion effects. Eur Polym J 71:534-546

Bhatia A, Gupta RK, Bhattacharya SN, Choi HJ (2007) Compatibility of biodegradable poly (lactic acid) (PLA) and poly (butylene succinate) (PBS) blends for packaging application. Korea-Aust Rheol J 19:125-131

Dennis Jr JE, Schnabel RB, Numerical methods for unconstrained optimization and nonlinear equations (Classics in applied mathematics, 16). Society for Industrial and Applied Mathematics (1996).

\section{Submit your manuscript to a SpringerOpen ${ }^{\circ}$ journal and benefit from:}

- Convenient online submission

- Rigorous peer review

- Immediate publication on acceptance

- Open access: articles freely available online

- High visibility within the field

- Retaining the copyright to your article

Submit your next manuscript at $>$ springeropen.com 